\title{
Characterization of Double Structure Tabular Microcrystals of Silver Halide Emulsions by Means of Electron Energy-Loss Spectroscopy, Zero-Loss Electron Spectroscopic Imaging and Energy-dispersive X-ray Microanalysis
}

\author{
Vladimir Oleshko $\left({ }^{1, *}\right)$, Renaat Gijbels $\left({ }^{1}\right)$, Willem Jacob $\left({ }^{2}\right)$, Frank Lakiere $\left({ }^{2}\right)$, André Van \\ $\operatorname{Dele}\left({ }^{2}\right)$, Eugeny Silaev $\left({ }^{3}\right)$ and Lilia Kaplun $\left({ }^{3}\right)$ \\ ( $\left.{ }^{1}\right)$ Department of Chemistry, University of Antwerp (UIA), B-2610 Wilrijk-Antwerpen, Belgium \\ (2) Department of Medicine, University of Antwerp (UIA), B-2610 Wilrijk-Antwerpen, Belgium \\ $\left({ }^{3}\right)$ Research Institute for the Photographic Industry, 125167 Moscow, Russia
}

(Received October 19, 1994; accepted February 13, 1995)

\begin{abstract}
Electron energy-loss spectroscopy (EELS) and zero-loss electron spectroscopic imaging (ZLESI) in conjunction with spot energy-dispersive X-ray microanalysis (EDX) were applied to the structural and chemical characterization of practical emulsion core $(\mathrm{AgBr})$-shell $\left(\mathrm{AgBr}_{1-x} \mathrm{I}_{x}\right)$ tabular microcrystals. The samples were cryocooled to minimize damage under the electron beam. Recorded energy-loss spectra of the crystals in the initial state and after a 2-minute development contained several low intensity bands, which were tentatively assigned to silver and halides, as well as bulk, surface and defect plasmons and interband transitions. Spatially resolved energy-loss spectra of silver specks on extractive carbon replicas of silver halide grains showed the presence of the delayed $\mathrm{Ag} \mathrm{M}_{4,5^{-}}$ edge at $400 \mathrm{eV}$. Results of EELS-microanalysis have been confirmed by EDX spot analysis of the same emulsion samples. The improved contrast in the ZLESI mode enabled the detection of dislocation grid and stacking faults on the tabular grains as well as the visualization of the morphology and defect structure of silver filaments formed during development of microcrystals. Zero-loss filtering was also used to obtain high-resolution point diffraction patterns of the crystals with low background, containing extra reflections at commensurate positions in between the Bragg reflections probably due to a number of defects in the shell region.
\end{abstract}

\section{Introduction}

Microcrystals of silver halide emulsions are commonly recognized as the best quantum detectors in photographic materials. Silver halide emulsions based on composite tabular crystals have some advantages over isometric ones, i.e. enhanced efficiency of quantum detection, reduced lightscattering, increased surface area, decreased silver deposition and thinner photographic layers.

$\left({ }^{*}\right)$ V. Oleshko is on leave from the Russian Academy of Sciences, N. N. Semenov Institute of Chemical Physics, 117421 Moscow, Russia. 
The wide application of novel types of silver halide emulsions based on multistructured tabular microcrystals stimulated interest in their characterization by means of different instrumental techniques [1-7].

Since the structural arrangement and the elemental distribution of $\mathrm{AgX}(\mathrm{X}=\mathrm{Cl}, \mathrm{Br}, \mathrm{I})$ affect drastically the photographic characteristics of emulsions, analytical electron microscope studies using energy-dispersive X-ray microanalysis (EDX) combined with various imaging modes [2, 5-7] and recently also electron energy-loss spectroscopy (EELS) and electron spectroscopic imaging (ESI) $[8,9]$ have been pursued with continued interest. The present work deals with some application of EELS and Zero-loss electron spectroscopic imaging (ZLESI) in conjunction with EDX for structural and analytical characterization of double structure silver halide tabular microcrystals of mixed composition.

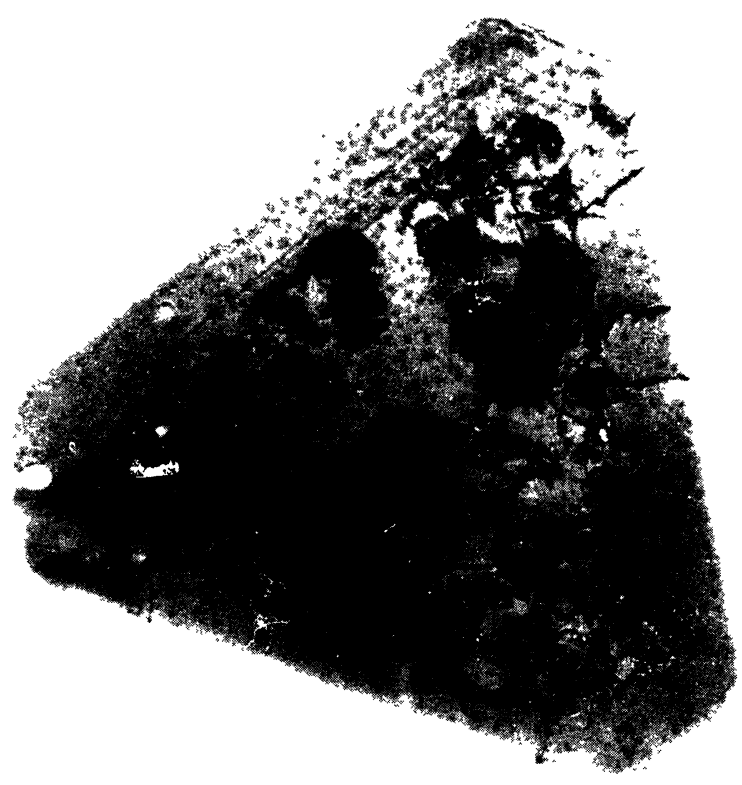

$100 \mathrm{~nm}$

Fig. 1. - ZLESI micrograph of a double structure silver bromiodide tabular microcrystal.

\section{Experimental}

Objects of study were practical core-shell tabular microcrystals synthesized by a controlled doublejet of $\mathrm{AgNO}_{3}$ and $\mathrm{KX}(\mathrm{X}=\mathrm{Br}, \mathrm{I})$. Crystals of $1-7 \mu \mathrm{m}$ in diameter contained $\mathrm{AgBr}$ cores with 


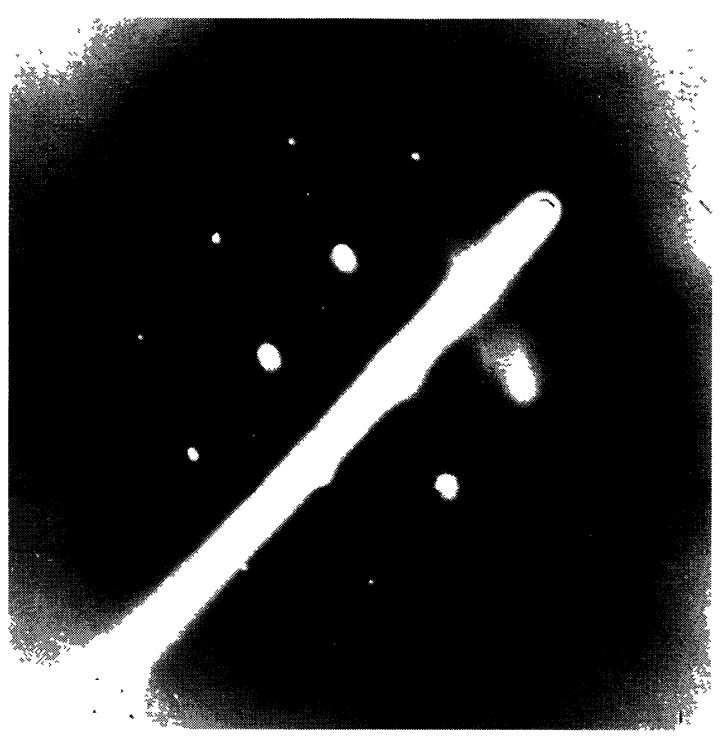

Fig. 2. - Inverted ZLSSAED pattern of a tabular emulsion crystal, [111] zone.

$\%$

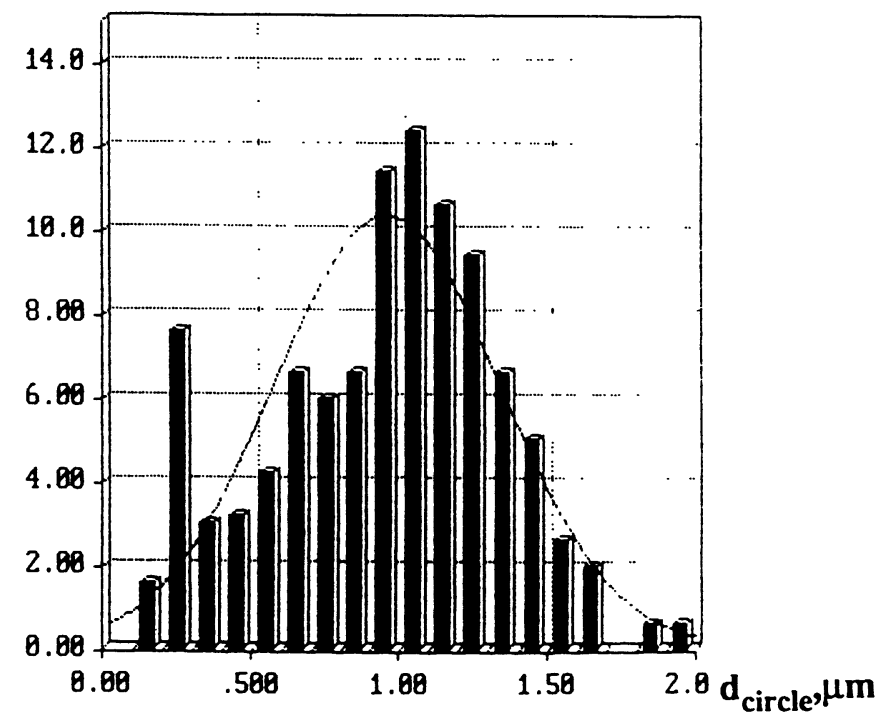

Fig. 3. - Histogram on size distribution of microcrystals, $d_{\text {circle }}, \mu \mathrm{m}$. A gaussian curve with the parameters of the given distribution is shown by the continuous line.

sizes of $0.5-5 \mu \mathrm{m}$ and $\mathrm{Ag}(\mathrm{Br}, \mathrm{I})$ shells containing $5 \mathrm{~mol} . \%$ of AgI. Deposition of the grains on thin carbon film-supports and carbon replicas technique described elsewhere [10] were used to prepare samples for electron microscope studies. 


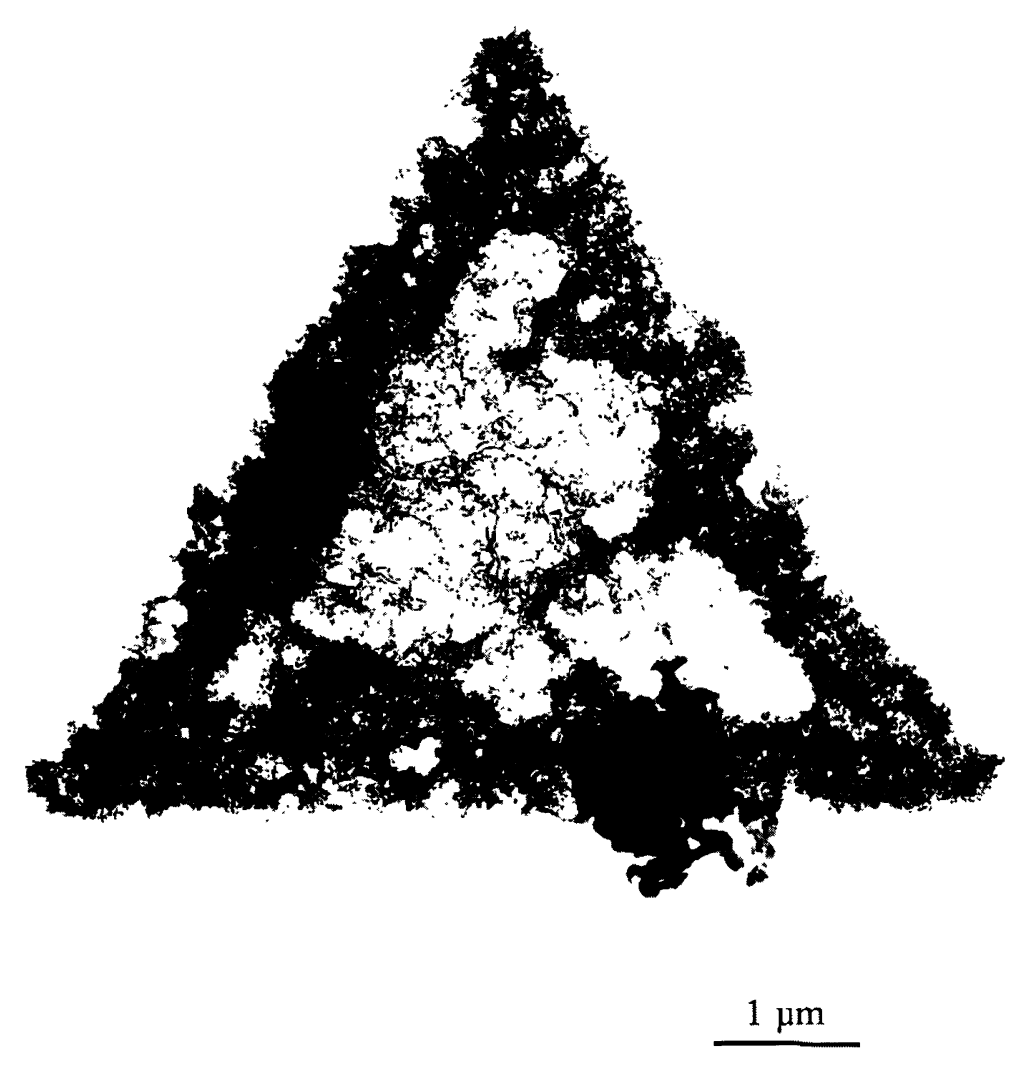

Fig. 4. - ZLESI micrograph of a tabular crystal after a 2-minute treatment with the Kodak developer D-19.

EELS/ZLESI observations were performed using a Zeiss EM 902 electron microscope with an integrated prism-mirror-prism energy filter of the Castaing-Henry type at an acceleration voltage $80 \mathrm{kV}$. Energy-loss spectra were acquired over a range of $0-2000 \mathrm{eV}$ with the aid of a photomultiplier serial detector system interfaced to a PC/AT for automatic data acquisition. The optimal signal-to-noise ratios of the elements of interest were obtained with an objective aperture of $60 \mu \mathrm{m}$ and with a filter entrance aperture of $100 \mu \mathrm{m}$ acting as a selected-area aperture. The analyses and observations of the crystals were performed at magnifications of $12,000-140,000$, corresponding to a selected area from about $800 \mathrm{~nm}$ to $20 \mathrm{~nm}$ in diameter. The estimated energy resolution was $1.5-2.0 \mathrm{eV}$, based on the measured full-width a half-maximum (FWHM) of the zero-loss peak. A top-entry cryostage cooled with liquid nitrogen was applied to minimize sample damage under electron beam irradiation.

A JEOL 1200EX electron microscope equipped with a scanning attachment and a TRACOR NORTHERN TN5500 EDX-system were used. The spot EDX-microanalysis was performed in the scanning transmission mode at $80 \mathrm{kV}$ accelerating voltage. The temperature of the specimen was kept at $t=-167^{\circ} \mathrm{C}$ by the use of a Gatan Model 636 tilt cooling holder in order to minimize silver halide crystal damage and drift under electron bombardment. It has been shown earlier [6] that at this temperature the X-ray count rates for the elements in silver halide can be considered as constant in the spot analysis mode. A KONTRON IBAS-2000 image analysis system is applied to estimate the size and thickness of distributions of the microcrystals. 


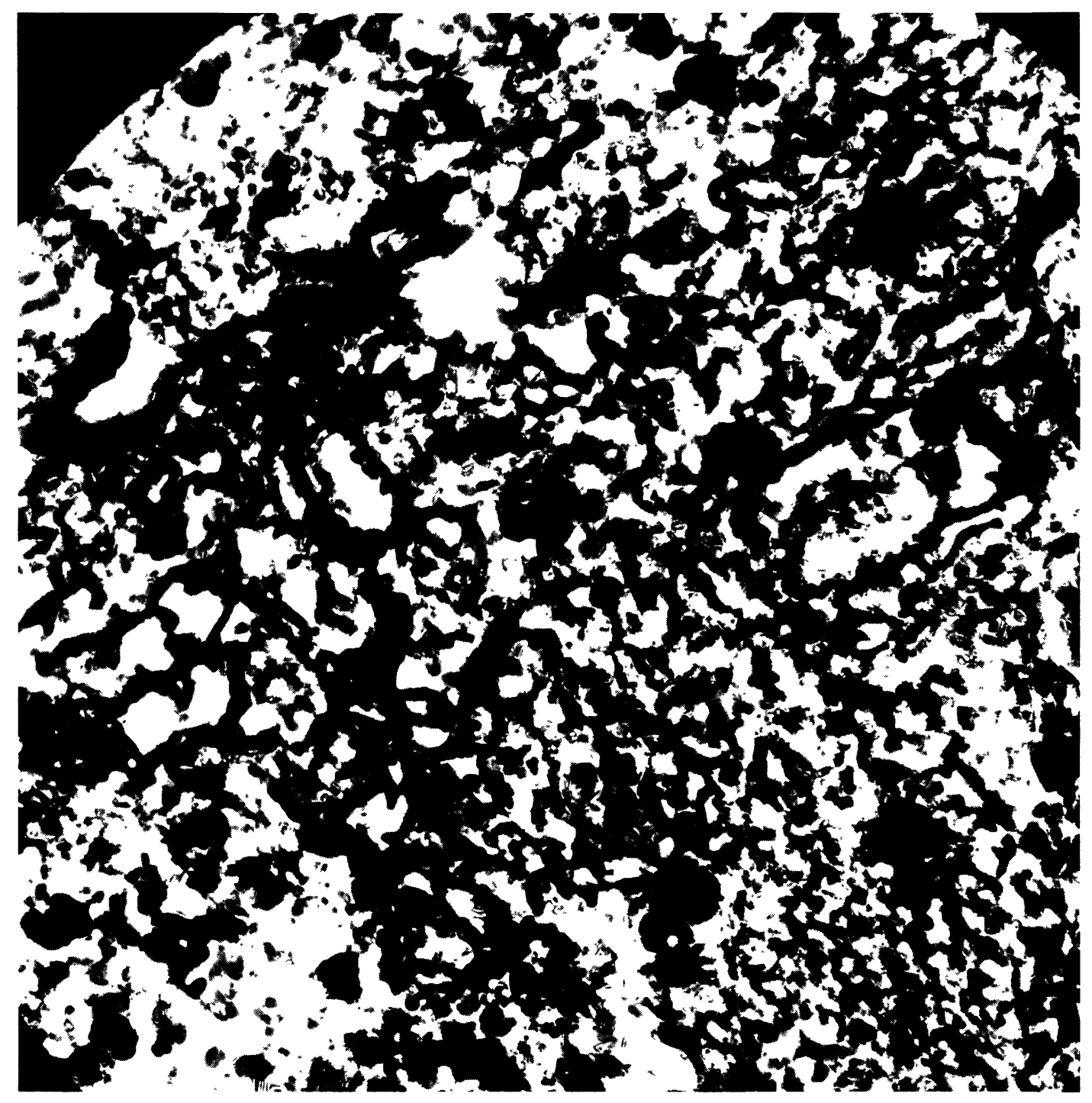

$100 \mathrm{~nm}$

Fig. 5. - ZLESI micrograph of a branched web-like network in the central part of the crystal.

\section{Zero-Loss Spectroscopic Imaging}

The morphology and crystallography of different types of grains and lattice defects have been studied in core-shell type silver halide tabular crystals mainly by means of conventional electron microscopy (CTEM) and scanning electron microscopy $[1,4,10]$. A ZLESI image of a double structure tabular crystal is presented in Figure 1. The grain consists of an $\mathrm{AgBr}$ core surrounded by a uniformly mixed $\mathrm{Ag}(\mathrm{Br}, \mathrm{I})$ shell. Most of the observed tabular grains had a hexagonal shape 


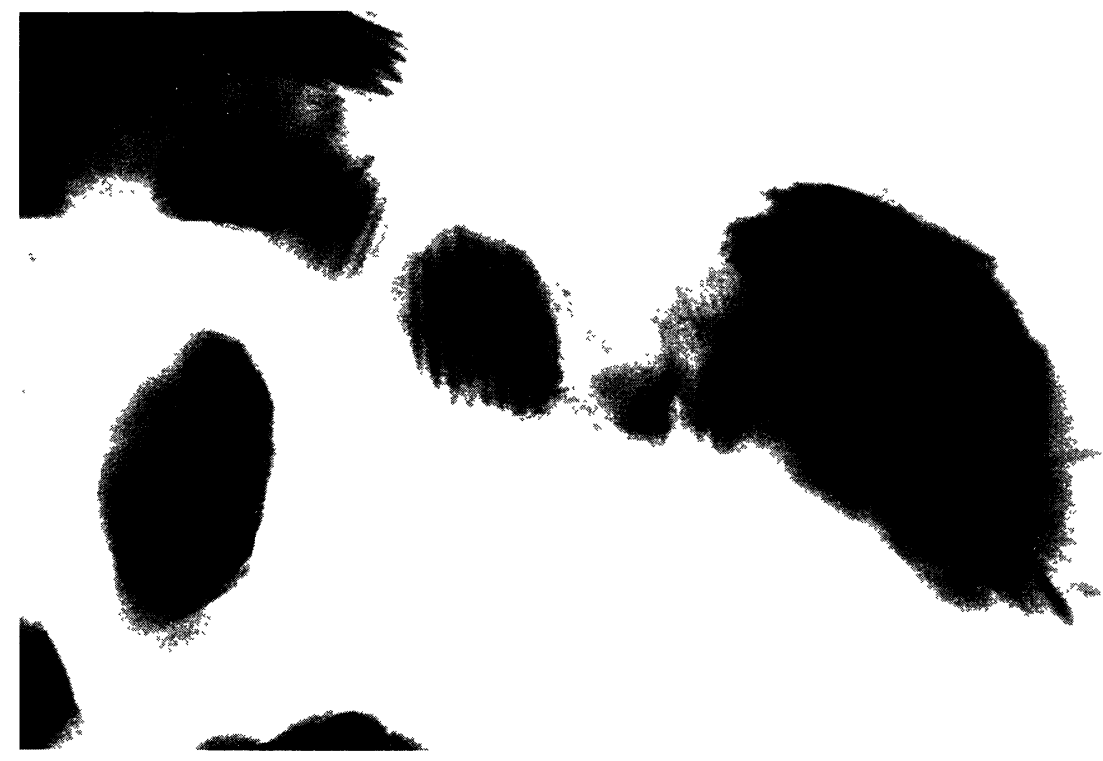

$10 \mathrm{~nm}$

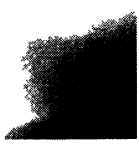

Fig. 6. - ZLESI image of Moire fringes with a period of about $4.5 \mathrm{~nm}$ due to overlap of two thin silver grains.

caused by the lateral growth along the $\{11 \overline{1}\}$ planes inclined over an angle of $70^{\circ} 32^{\prime}$ with respect to the (111) surfaces and only a few percent were isometric or rod-like. The high surface-tovolume ratio of tabular microcrystals points to the importance of surface defects and the diffuse ionic space-charge layer in the formation of the latent image and in the development process [11]. Iodide incorporated in the $\operatorname{AgBr}_{1-x} I_{x}$ shell introduces strong lattice distortions with atom displacements perpendicular to the core-shell interface. As a consequence, the cationic sublattice binding energy decreases and various types of surface and internal defects are formed.

An improved contrast in the ZLESI mode as compared with the CTEM mode enabled not only to detect more clearly numerous edge and random dislocations attached to photolytic sites and complicated defects localized near the interface between the core and the shell, but also to improve resolution of $\{11 \overline{1}\}$ stacking faults in the shell region parallel to $\{11 \overline{2}\}$ grain edges. The presence of $\{11 \overline{1}\}$ stacking fauths is supported by observations of extra reflections at commensurate positions in between the Bragg reflections revealed in diffraction patterns (see Fig. 2), perhaps, due to a large number of crystallographic defects in the shell region parallel to the grain edges [4].

Figure 3 presents a size distribution of emulsion crystals (one of them was shown in Fig. 1) with the mean diameter $0.95 \pm 0.03 \mu \mathrm{m}$ and the mean thickness $107 \pm 4 \mathrm{~nm}$ determined by means of replicas technique. An approximate evaluation using EELS log-ratio technique with accuracy about $\pm 20 \%$ [12] gave the mean crystal thickness value $98 \mathrm{~nm}$ and was in satisfactory agreement with measurements on grain replicas.

Observations of grain extractive replicas showed that the formation of the photolytic specks occurred mainly on defects localized in the core region and near the interface between the core and the shell where the concentration of interstitial silver cations was increased. 


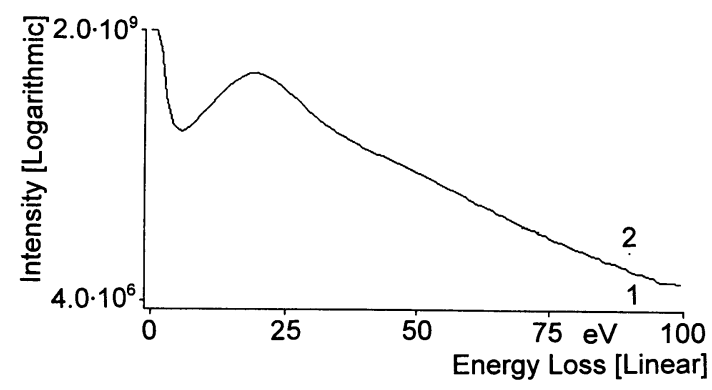

a

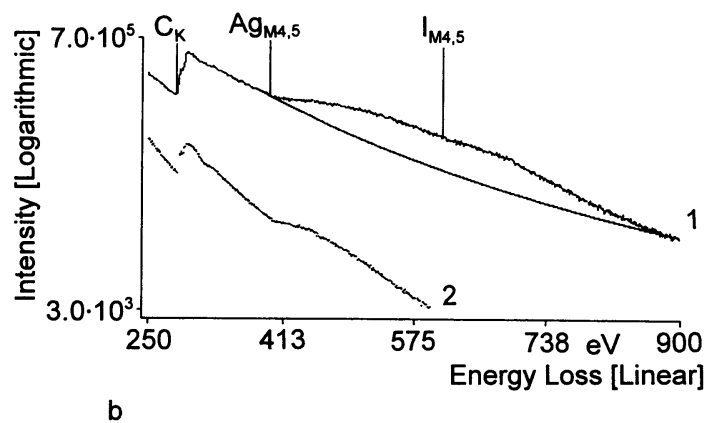

Fig. 7. - Energy-loss spectra of a tabular microcrystal at $t=-177^{\circ} \mathrm{C}$ (curve 1) and reference self-supported silver dendritic crystals (curve 2) recorded in the low-loss region (a) and in the core excitation region (b).

Development of the crystals during a 2-minute treatment with the Kodak developer D-19 was usually accompanied with an intensive etching of the core and resulted in the formation of a network of silver filaments preferentially in the core region. The development process in the shell regions of large crystals (see Fig. 4) occurred more slowly and etching was not so intensive because of the lower solubility of $\operatorname{AgBr}_{1-x} I_{x}$ as compared with pure AgBr. Due to the presence of a protective gelatin film adsorbed on the crystal surface the external shape of the grains did not change in the course of development. When the sizes of crystals became smaller, the difference in speed of development between the core and the shell decreased and the inhibition effect of iodide was almost absent.

In some cases, when there was no significant difference in iodide concentration between the core and the shell, the process proceeded with the same speed in both parts of the crystal. In the central part of the crystal, silver filaments and particles formed a branched web-like network (see Fig. 5). As was seen from CTEM and ZLESI images of the same area, zero-loss filtering improved the contrast and the resolution of numerous defects (stacking faults and dislocations) and grain boundaries. A filtered image presented in Figure 6 shows Moire fringes with a period of about $4.5 \mathrm{~nm}$ resulting from the overlap of two thin silver grains.

\section{Electron Energy-Loss Spectroscopy and Energy-Dispersive X-ray Microanalysis}

Energy-loss spectra of a tabular microcrystal (curve 1) and of reference self-supported silver dendritic crystals (curve 2) recorded in the low-loss region (a) and in the core excitation region (b) 

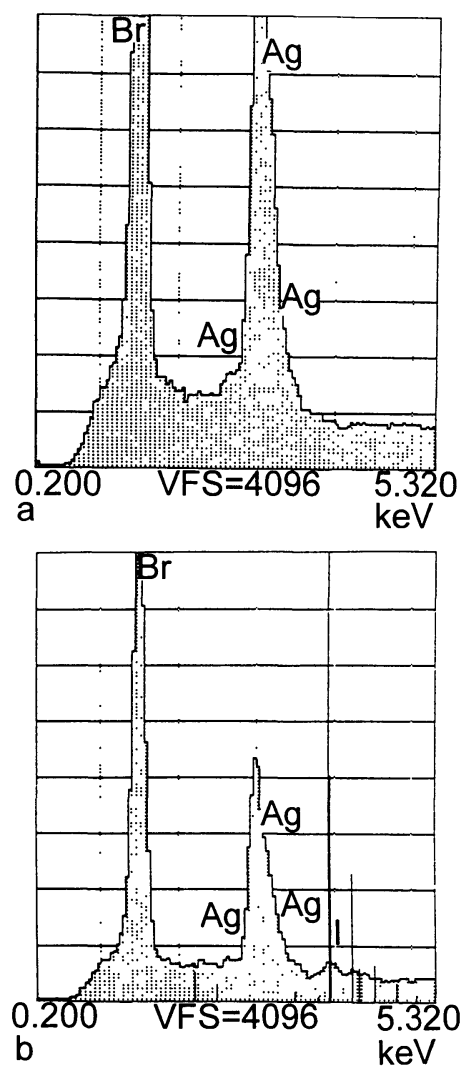

Fig. 8. - X-ray emission spectra recorded at selected points of a crystal core (a) and shell (b). KLM-markers denote the position of the I L-series.

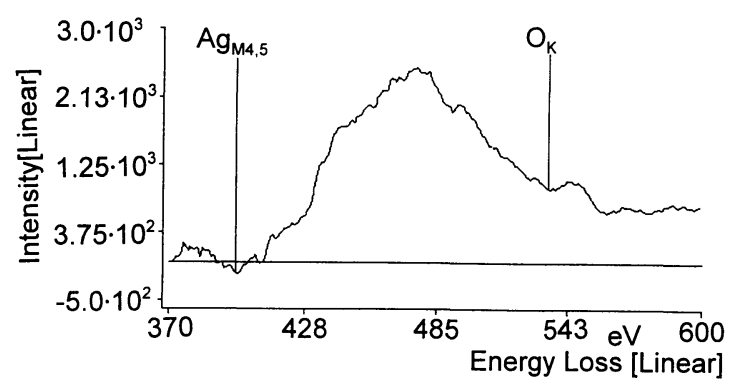

Fig. 9. - The net $\mathrm{Ag} M_{4}$, 5 -edge obtained by subtracting the background from an energy-loss spectrum of an $80 \mathrm{~nm}$-sized silver speck on the carbon replica of a tabular microcrystal followed by smoothing of the signal with the Savitsky-Golay filter of second $\operatorname{order}(n=2)$ and a width $p=23$.

are presented in Figure 7. The bulk plasmon peak at $24-26 \mathrm{eV}$ shown in Figure 7a sometimes exhibited a shift to low energy-losses and several weak features at $7-19 \mathrm{eV}$, probably concerned with the contribution of surface and defect plasmons. The fitted background (Fig. 7b, curve 1) 
was calculated to reveal positions of the low intensity elemental edges. The spectrum contained several bands, which probably can be attributed to iodide (I $M_{4,5}$-edge at $619 \mathrm{eV}$ ), silver (delayed $\mathrm{Ag} M_{4,5}$-edge at $400 \mathrm{eV}$ ) and carbon (C K-edge at $\left.284 \mathrm{eV}\right)$. Bromide edges $\left(\mathrm{Br} M_{4,5}\right.$-edge at $70 \mathrm{eV}$ and delayed $\mathrm{Br} I_{2}, 3$-edge at $1550 \mathrm{eV} \mathrm{[13])} \mathrm{were} \mathrm{not} \mathrm{clearly} \mathrm{detected,} \mathrm{perhaps,} \mathrm{due} \mathrm{to} \mathrm{low}$ intensities of the bands and high intensity of the background in the corresponding range of energy losses. The results of the assignment of the energy-loss spectra were compared with data of the EDX-microanalysis of the same emulsion samples.

$\mathrm{X}$-ray emission spectra recorded at selected points of a silver bromiodide grain (position 1, core (a) and position 2, shell (b)) are presented in Figure 8. X-ray spectra contained intensive bands of the $\mathrm{Ag} \mathrm{L}$-series $\left(\mathrm{Ag} \mathrm{L} \alpha_{1}\right.$ at $2.99 \mathrm{keV}$ and $\mathrm{Ag} \mathrm{L} \beta_{1}$ at $\left.3.15 \mathrm{keV}\right)$ and of the $\mathrm{Br} \mathrm{L}$-series $(\mathrm{Br} \mathrm{L} \alpha$ at $1.49 \mathrm{keV}$ and $\mathrm{Br} \mathrm{L} \beta_{3}$ at $\left.1.60 \mathrm{keV}\right)$. The $\mathrm{Br} \mathrm{K} \alpha$ line at $11.92 \mathrm{keV}$ and $\mathrm{Br} \mathrm{K} \beta_{1}$ line at $13.28 \mathrm{keV}$ (not shown in the spectra) were also detected. The low intensity I L-series (I L $\alpha$ at $3.92 \mathrm{keV}$ and I $L \beta_{1}$ at $4.22 \mathrm{keV}$ ) was detected in the case of the shell region (spectrum b). Some decrease of the spectral intensity in spectrum $b$, as compared with spectrum a, might be due to an edge effect during X-ray generation. As a rule, EDX confirmed the presence of iodide in the crystal shells.

Figure 9 shows the net $\operatorname{Ag} M_{4,5}$-edge was obtained by subtracting the background from an initial energy-loss spectrum of a $80 \mathrm{~nm}$-sized silver speck on a grain replica followed by subsequent smoothing of the signal. The smoothed spectrum indicated the presence of oxygen $(\mathrm{O} K$-edge at $533 \mathrm{eV}$ ). Energy-loss spectra of developed crystals revealed the weak I $N_{4}, 5$-edge at about $50 \mathrm{eV}$ and the $\mathrm{Br} M_{4,5}$-edge at $68 \mathrm{eV}$ and significantly increased intensity of the $\mathrm{Ag} M_{4}, 5$-edge due to formation of thin silver filaments. Comparison of the spectra of the core region and the shell region of the same crystal showed clearly the appearance of the I $M_{4}, 5$-edge at $619 \mathrm{eV}$ due to its accumulation in the core region during development. Multiple plasmon losses at 4, 7, 16, 24 , and $32 \mathrm{eV}$, respectively, were detected in the case of the conductive network of thin silver filaments formed in the central part of the crystal core. These multiple plasmon losses may be due to oscillations of the imaginary part of the energy-loss function $\operatorname{Im}[-1 / \varepsilon(E)]$ caused by interband transitions $[13,14]$. On the contrary, in the case of the crystal shell an assymetric peak at $21.5 \mathrm{eV}$ probably due to bulk plasmons arisen in silver halide residues with iodide and the contribution of surface and defect plasmons dominated in the low-loss spectrum.

\section{Conclusions}

EELS/ZLESI in conjunction with EDX spot microanalysis have been applied for combined structural and analytical characterization of practical emulsion core $(\mathrm{AgBr})$-shell $\left(\mathrm{AgBr}_{1-x} \mathrm{I}_{x}\right)$ tabular microcrystals. Recorded energy-loss spectra of the crystals in the initial state and after a twominute treatment with the Kodak developer D-19 contained several low intensity bands, which could be assigned to silver and halides, and bulk, surface and defect plasmons and interband transitions. Results of EELS-microanalysis have been confirmed by EDX spot analysis of the same emulsion samples.

The improved contrast in the brightfield ZLESI mode enabled to detect numerous edge and random dislocations attached to photolytic sites, complicated defects localized near an interface between the core and the shell and stacking faults in the shell of the crystals. It was in agreement with observations of extra reflections at commensurate positions in between the Bragg reflections revealed in diffraction patterns, and probably due to a number of defects in the shell region. The morphology and defect structure of silver filaments formed during development of microcrystals has also been studied using zero-loss filtering. 


\section{Acknowledgements}

This work was supported by the Belgian National Foundation for Scientific Research (NFWO) and by the Federal Services for Scientific, Technical and Cultural Affairs (DWTC/SSTC) of the Prime Minister's Office through IUAP-III, Convention-49.

\section{References}

[1] Maskasky J.E., J. Imag. Sci. 31 (1987) 15.

[2] Gao X.-L., Gijbels R., Nys B., Jacob W., Gilliams Y., J. Imag. Sci. 33 (1989) 87.

[3] Maternaghan T.J., Falder C.J., Levi-Setti R. and Shabala J.M., J. Imag. Sci. 34 (1990) 58.

[4] Goessens C., Schryvers D., Van Landuyt J., Amelinckx S., Verbeeck A. and De Keyzer R., J. Cryst. Growth 110 (1991) 930.

[5] Geuens I., Gijbels R., Jacob W., Verbeeck A. and De Keyzer R., J. Imag. Sci. Techn. 36 (1992) 534.

[6] Black D.L. and Timmons J.A., Imag. Sci. Techn. 38 (1994) 10.

[7] Lavergne J.L., Gimenez C., Friour G. and Martin J.M., In Proc. Intern. Congr. Phot. Sci. ICPS'94/IS7T's 47th Ann. Conf. Vol. 1 (Rochester, New York, 1994) p. 31.

[8] Lavergne J.L., Gimenez C., Friour G. and Martin J.M., In Proc. 13th Intern. Congr. Electr. Microscopy, B. Jouffrey and C. Colliex Eds., Vol. 1 (Les Editions de Physique, Les Ulis, France, 1994) p. 629.

[9] Oleshko V. and Alfimov M., Imag. Sci. Techn. 38 (1994) 162.

[10] Maskasky J.E., J. Imag. Sci. 31 (1987) 93.

[11] Tan Y.T., J. Chem. Soc. Faraday Trans. 2 (1989) 457.

[12] Malis T., Cheng S.C. and Egerton R.F., J. Electr. Microsc. Tech. 8 (1988) 193.

[13] Egerton R.F., Electron Energy-Loss Spectroscopy in the Electron Microscope (Plenum Press, New York and London, 1986).

[14] Raether H., Excitation of Plasmons and Interband Transitions by Electrons, Springer Tracts in Modern Physics, Vol. 88 (Springer-Verlag, New York, 1980). 